\title{
A New Method for In Vivo Targeted Gene Transfer into Oligodendrocytes using Adenoviral and HIV Vectors
}

\author{
Koichi Miyake $^{1 *}$, Noriko Miyake ${ }^{2}$ and Takashi Shimada ${ }^{2}$ \\ ${ }^{1}$ Department of Gene Therapy, Nippon Medical School, Tokyo, Japan \\ ${ }^{2}$ Department of Biochemistry and Molecular Biology, Nippon Medical School, Tokyo, Japan
}

*Corresponding author: Koichi Miyake, Department of Gene Therapy, Nippon Medical School, 1-1-5 Sendagi, Bunkyo-ku, Tokyo

113-8602, Japan

\section{ARTICLE INFO}

Received: 蔧 October 10, 2021

Published: 幽 October 20, 2021

Citation: Koichi Miyake, Noriko Miyake, Takashi Shimada. A New Method for In Vivo Targeted Gene Transfer into Oligodendrocytes using Adenoviral and HIV Vectors. Biomed J Sci \& Tech Res 39(3)2021. BJSTR. MS.ID.006306.

Keywords: Targeted Gene Transfer; Oligodendricytes; Adenoviral Vector; HIV Vector; Non-Dividing Cells

\begin{abstract}
To achieve oligodendrocyte (OL)-specific gene transfer, we applied a two-step method in which target cells were first engineered to express CD4 in a tissue-specific manner using adenoviral (Ad) vectors and were then transduced with human immunodeficiency viral (HIV) vectors. Primary cultures of mixed rat brain cells were initially incubated with an Ad vector harboring the human CD4 gene under the control of the OL-specific myelin basic protein promoter and a second Ad vector harboring the CXCR4 gene. The cells were subsequently incubated with an HIV vector harboring the enhanced green fluorescent protein (EGFP) gene. With this protocol, only OLs previously transduced with the Ad vectors were transduced with the HIV vector. Furthermore, when we injected the Ad and HIV vectors into the brains of adult rats, only OLs were transduced. These results suggest that a combination of Ad and HIV vectors could be useful for development of neuronal cell-specific gene transfer protocols. And because both Ad and HIV vectors can transduce non-dividing cells, this method may enable the in vivo targeting of any type of non-dividing cells for gene transfer just by choosing the appropriate promoter.
\end{abstract}

Abbreviations: Ad: Adenoviral; HIV: Human Immunodeficiency Viral; EGFP: Enhanced Green Fluorescent Protein; MBP: Myelin Basic Protein; TU: Transducing Unit; MOI: Multiplicity of Infection; FACS: Fluorescence Activated Cell Sorting; PBS: Phosphate Buffered Salts

\section{Introduction}

Viral vectors such as retrovirus, adenovirus (Ad), lentivirus, and adeno-associated virus (AAV) are widely used in clinical gene therapy protocols as vehicles for the delivery of genes into mammalian cells [1-5]. One advantage of these vectors is their ability to transduce a wide range of cell types, but this lack of specificity is also a distinct disadvantage, especially for in vivo gene transfer. This is because transduction of both target and nontarget cells results in massive wastage of the vector, and stable gene transfer into non-target cells by retroviral vectors may increase the likelihood of insertional mutagenesis in the transduced cells [6,7]. An efficient technique for targeted gene transfer would thus be a highly desirable.
Several strategies for targeting cells for gene transfer have been reported. One approach is to achieve transcriptional targeting using a tissue-specific promoter $[8,9]$. With this strategy, however, non-target cells are also transduced, even though the promoter is silent, resulting in massive wastage of the vectors. Another strategy is receptor-mediated targeting. For example, many investigators have been able to achieve targeted retroviral gene transfer through modification of the vector particles using single chain antibody fragments $[10,11]$ and ligand molecules $[12$, $13]$, and by using pseudo type viruses $[14,15]$. The low efficiency of gene transfer is a serious disadvantage of this approach, however. To overcome these problems, we developed a novel strategy for 
cell targeting based on tissue-specific expression of an ecotropic retroviral receptor gene using Ad and ecotropic retroviral vectors [16]. With this approach, we achieved efficient targeted retroviral transduction through Ad-mediated, tissue-specific expression of a retrovirus receptor. Unfortunately, non-dividing cells could not be transduced using this method because cell division is required for the retrovirally mediated gene transfer [17].

Human immunodeficiency virus (HIV)-based retroviral vectors have several interesting features that make it potentially useful for targeted gene transfer. Because CD4 antigen is a major receptor for HIV entry, HIV vectors transduce only human CD4-expressing cells [18]. In addition, the HIV vector, itself, provides receptormediated targeting based on the natural tropism of viruses, and it can transduce non-dividing cells [19]. To make full use of these features of the HIV vector, we developed a new method that expands the host range of the HIV vector through a two-step gene transfer protocol [20]. Using this protocol, we were able to stably transduce such non-dividing cells as neurons and terminally differentiated macrophages [21], suggesting a combination of $\mathrm{Ad}$ and HIV vectors is potentially useful for transduction of a variety of non-dividing cell types. To further develop this strategy for targeted gene transfer into non-dividing cells, in the present study we constructed an Ad vector expressing the CD4 gene under the control of the oligodendrocyte (OL)-specific myelin basic protein (MBP) promoter [22,23]. We then tested whether OL-specific gene transfer could be achieved using Ad and HIV vectors, and whether this new method could be used for targeting gene transfer into nondividing cells in vivo.

\section{Materials and Methods}

\section{Cells}

Cos, 3T3, HEK293 and CD4H (CD4+ HeLa) cells [20] were grown in Dulbecco modified Eagle medium (DMEM) supplemented with $10 \%$ heat-inactivated fetal bovine serum (FBS) and 100 units/ml penicillin and $100 \mu \mathrm{g} / \mathrm{ml}$ streptomycin (GIBCO-BRL, Gaithersburg, MD) at $37{ }^{\circ} \mathrm{C}$ in $5 \%$ carbon dioxide. The CG4 OL cell line was maintained and differentiated as described previously in growth medium or differentiation medium [24]. Primary cultures of mixed rat brain cells were prepared as previously described [25] with some modification. Briefly, embryonic brains were minced and dissociated by pipetting after treatment with $0.25 \%$ trypsin. After low speed centrifugation (500rpm, 3min) to remove the debris and filtration through a $70 \mathrm{~mm}$ filter, the cells were grown in MEM/F12 medium (GIBCO-BRL, Gaithersburg, MD) supplemented with $10 \%$ FBS to obtain a mixed glial culture.

\section{Production of Ad and HIV vectors}

A replication-defective Ad vector was generated using the Saito method [20]. An Ad vector containing the CD4 gene under the control of the MBP promoter (kindly provided by Dr. Ikenaka, National Institute for Physiological Sciences, Okazaki, Japan) was constructed by inserting the expression unit containing the MBP promoter (1.3-kb HindIII fragment) [26], the coding sequence of CD4, and the rabbit ß-globin polyadenylation signal into pAdex1w [27]. Another Ad vector containing the CXCR4 gene [28,29] was constructed by inserting the coding sequence of CXCR4 (kindly provided by Dr. Matsushima, University of Tokyo, Tokyo, Japan) into pAdex1wCA [27]. These constructs and EcoT221 digested Ad DNA terminal protein complex were introduced together into HEK293 cells. The recombinant Ad, Ad/MBPCD4 and Ad/ CAGCXCR4 were isolated, purified and concentrated through cesium chloride gradient centrifugation. The titers of Ad/MBPCD4 and Ad/CAGCXCR4 were $3 \times 10^{10}$ and $1 \times 10^{10} \mathrm{PFU} / \mathrm{ml}$, respectively. An HIV vector carrying the enhanced green fluorescent protein (EGFP) gene (HXGFP) was generated by transient transfection of Cos cells with packaging (pCGPE) and vector (pHXGFP) plasmids as described previously [30]. Two days after transfection, the viruscontaining supernatant was concentrated by ultrafiltration using a CENTRIPREP 50 (Millipore Corporation, Bedford, MA) [30]. The biological titer of the concentrated HIV vector was approximately $10^{8}$ transducing units (TU)/ml when $\mathrm{CD} 4 \mathrm{H}$ cells were used as the target cells.

\section{Targeted Gene Transfer into OLs}

To achieve OL-specific gene transfer, we applied the two-step gene transfer method using Ad and HIV vectors as described previously [20]. In brief, rat brain primary mixed cultures were incubated with Ad/MBPCD4 and Ad/CAGCXCR4 for 60min at an MOI of 10. After two days of culture in complete medium, the cells were incubated with HXGFP for $48 \mathrm{~h}$ at an MOI of 100. For in vivo targeted gene transfer, 6- to 8-week-old Fischer 334 female rats (Japan Clea Co. LTD., Tokyo, Japan) were anesthetized with ketamine $(100 \mathrm{mg} / \mathrm{kg})$ and nembutal $(50 \mathrm{mg} / \mathrm{kg})$ and placed into a stereotaxic frame. Thereafter, the skull was exposed, a hole was drilled over the injection site $(0.7 \mathrm{~mm}$ anterior to bregma, $2.0 \mathrm{~mm}$ lateral, $4.0 \mathrm{~mm}$ vertical) [31], and $1 \mu \mathrm{l}$ of Ad/MBPCD4 and Ad/CAGCXCR4 was infused over period of $10 \mathrm{~min}$ using a Hamilton syringe with a 26 gage needle. Three days later, $2 \mu$ l of HXGFP were infused into the same site. As a control, Ad/CAGLacZ (kindly provided by Dr. Saito, University of Tokyo, Tokyo, Japan) plus Ad/CAGCXCR4 and HXGFP or only HXGFP were injected. All experiments involving animals were conducted according to the institutional guidelines of the Nippon Medical School.

\section{Flow Cytometric Analysis}

Expression of CD4 or CXCR4 was analyzed by flow cytometry (FACS Calibur, Becton Dickinson, Franklin Lakes, NJ) after staining with fluorescein isothiocyanate (FITC)-conjugated anti-human CD4 or phycoerythrin (PE)-conjugated anti-human CXCR4 (BD Pharmingen, San Diego, CA). 


\section{Immunocytochemistry and Immunohistochemistry}

To identify OLs, we used anti-carbonic anhydrase II (CAII) and anti-MBP antibodies (Dako, Hamburg, Germany) as described previously [32,33]. The transduced mixed rat brain cells were fixed in $4 \%$ paraformaldehyde for $15 \mathrm{~min}$ at room temperature. After washing three times with PBS, the cells were incubated with anti-CAII antibody and normal rabbit serum (Dako, Hamburg, Germany) for $2 \mathrm{~h}$ at room temperature. The cells were then washed three times in PBS containing 0.03\% Triton X and exposed to Texas Red-conjugated secondary antibody with normal rat serum (Dako, Hamburg, Germany). The stained cells were examined under a IX/70 inverted fluorescence microscope (Olympus, Tokyo, Japan) or analyzed by FACS Calibur. To analyze in vivo transduction of rat brain, 5 days or 3 months after injection of HXGFP, the rats were anesthetized and perfused with 4\% paraformaldehyde. The brains were then removed and transferred to PBS solution containing 20\% sucrose and stored overnight at $4{ }^{\circ} \mathrm{C}$. The next day, $40-\mu \mathrm{m}$-thick tissue sections were cut using a cryostat, after which the sections were blocked for $1.5 \mathrm{~h}$ in PBS containing $10 \%$ normal rabbit serum with $0.03 \%$ Triton $\mathrm{X}$ and incubated with anti-CAII or antiMBP antibody overnight at $4{ }^{\circ} \mathrm{C}$. The sections were then washed three times in PBS containing $0.03 \%$. Triton $\mathrm{X}$ and incubated with rhodamine (TRITC)-conjugated secondary antibody (Dako, Hamburg, Germany). After immunostaining, the tissue sections were mounted on slides and visualized and photographed using a confocal laser-scanning microscope (Leica TCSSP, Heidelberg, Germany) as described previously [32].

\section{Results}
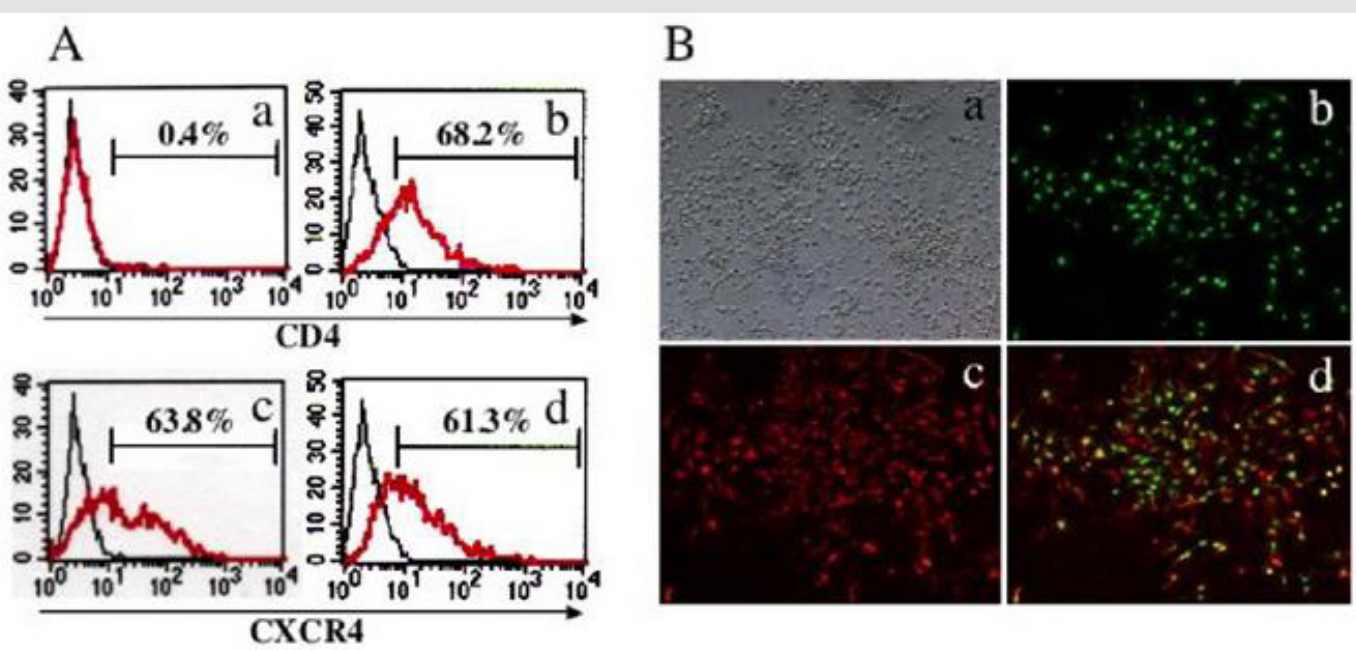

Figure 1: OL-specific CD4 expression by Ad/MBPCD4.

A. FACS analysis of Ad/MBPCD4 transduced cells.

- The upper panel shows a FACS analysis of Ad/MBPCD4-transduced 3T3 (a) and CG4 (b) cells

- As a control, the lower panel shows Ad/CAGCXCR4-transduced 3T3 (c) and CG4 (d)cells.

B. Immunocytochemical analysis of mixed primary rat brain cells transduced with Ad/MBPCD4.

- (a) Bright-field image.

- (b and c) Fluorescence microscopic images of cells double immuno stained with anti-CD4 (FITC) (b)

and anti-CAII (Texas-Red) (c)

- (d) Merged image combining b with c.

Oligodendrocytes, which are known to be myelin-forming cells, are an important target cell for gene therapy aimed at treating such demyelinating diseases as multiple sclerosis and metachromatic leukodystrophy. Because OLs are postmitotic, they cannot be transduced using moloney murine leukemia virus-based retroviral vectors. On the other hand, both Ad and HIV vectors are able to transduce non-dividing cells. Furthermore, Ad vectors with the MBP promoter have proven useful for OL-specific gene expression both in vitro and in vivo [33]. To assess OL-specific expression, we first examined CD4 and CXCR4 expression in 3T3 and CG4 cells transduced with Ad/MBPCD4 or Ad/CAGCXCR4. Expression of CXCR4 was detected in both cell types transduced with $\mathrm{Ad} /$ 
CAGCXCR4, while expression of CD4 was detected only in Ad/ MBPCD4-transduced CG4 cells (Figure 1A). We then confirmed the OL-specific expression of CD4 using primary mixed rat brain cell cultures. Among the different cell types transduced with $\mathrm{Ad} /$ MBPCD4 only CAII-positive cells expressed CD4 (Figure 1B), indicating that Ad/MBPCD4 selectively mediated expression in OLs. We next evaluated the utility of our two-step gene transfer system using mixed rat brain cells first incubated with Ad/MBPCD4. And because non-human cells do not express CXCR4, which is a coreceptor for HIV, these primary cells were also incubated with Ad/ CAGCXCR4. Two days later, the cells were incubated with HXGFP and, after an additional 2 days, they were stained with anti-CAII or
anti-MBP antibody. We found that all EGFP-positive cells were also CAII-positive (Figure 2A). In addition, FACS analysis showed that only MBP-positive cells could be transduced with HXGFP (Figure 2B), indicating that OLs first transduced with Ad vectors were then selectively transduced with the HIV vector. Thus transient selective expression of CD4 molecules using Ad/MBPCD4 is apparently sufficient to render OLs susceptible to HIV-mediated gene transfer. To then determine whether this new method could be used in vivo to target gene transfer into OLs, we injected the Ad and HIV vectors into the brains of adult rats. Five days after injection of the HIV vector, some of the rats were fixed and their brains were examined using confocal laser-scanning microscopy.
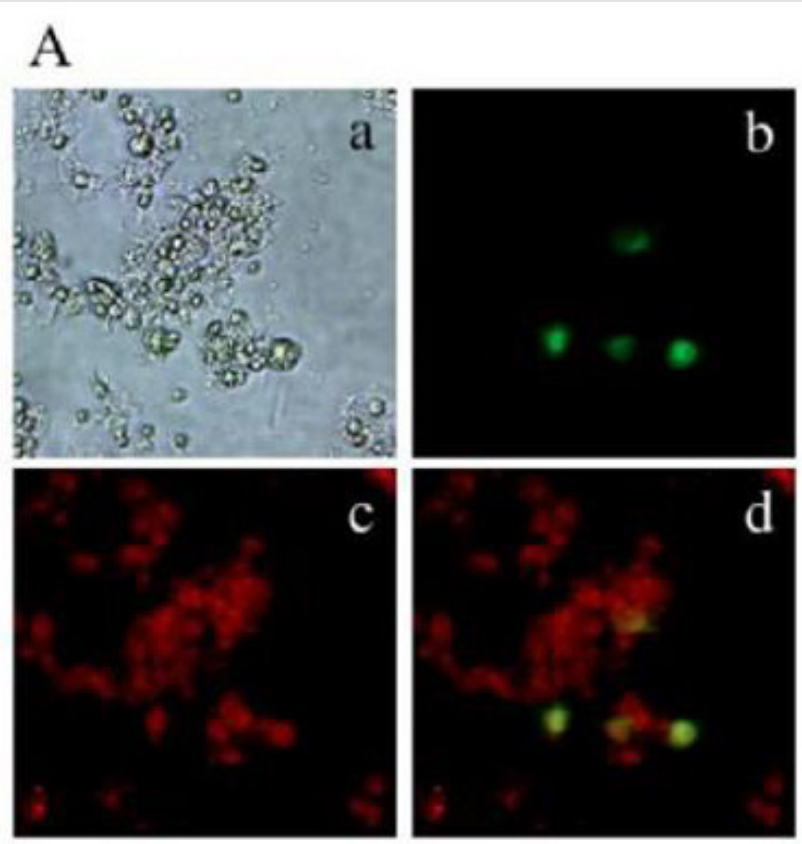

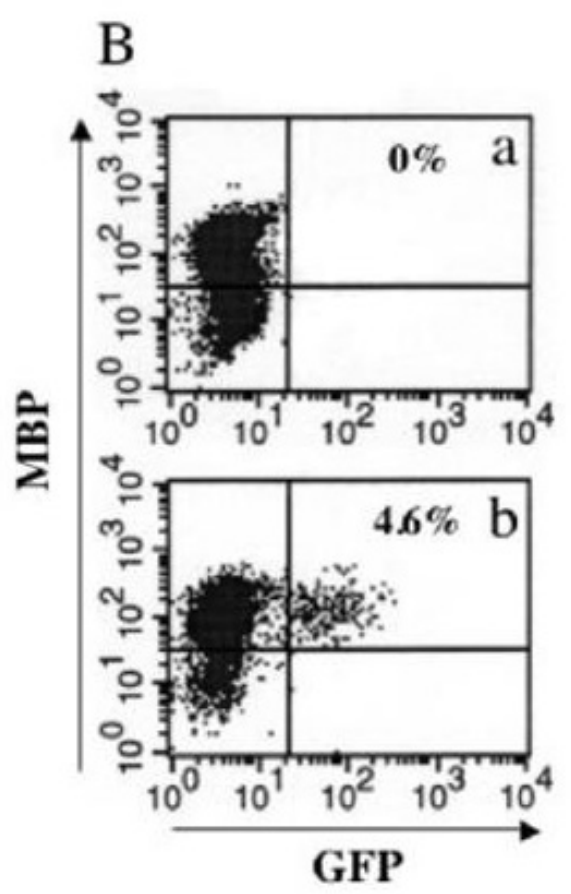

Figure 2: OL-specific transduction using a two-step gene transfer method.

A. Immunocytochemical analysis of primary rat brain cells transduced using a two-step gene transfer method.

- (a) Bright-field image.

- (b and c) Fluorescence microscopic images of GFP (b) and CAII (Texas-Red) (c)

- (d) Merged image combining (b) with (c).

B. FACS analysis of primary rat brain cells transduced by two-step gene transfer method.

- FACS analysis of primary rat brain cells transduced with Ad/CAGLacZ (a) or Ad/MBPCD4 (b) plus Ad/CAGCXCR4 and HXGFP. After immunostaining with anti-MBP, the cells were analyzed by flow cytometry.

EGFP-positive cells were not be detected in rats injected with the control vector Ad/CAGLacZ plus Ad/CAGCXCR4 and HXGFP or with HXGFP alone (data not shown). On the other hand, we were able to detect EGFP-positive cells in rats injected with Ad/ MBPCD4 plus Ad/CAGCXCR4 and HXGFP (Figure 3A). Moreover, immunohistochemical staining using anti-CAII and anti-MBP (not shown) antibodies revealed that all of the EGFP-positive cells were also CAII-positive (Figures 3B \& 3C) and MBP-positive, indicating that only OLs were transduced using this two-step gene transfer method. In some cases, the rats were not sacrificed and analyzed until 3 months after vector injection. Notably, the results obtained 3 months after vector injection were nearly the same as those obtained 5 days after vector injection (Figures 3D-3F). This strongly suggests we were able to integrate the transgene into the host genome and obtain sustained transgene expression. 


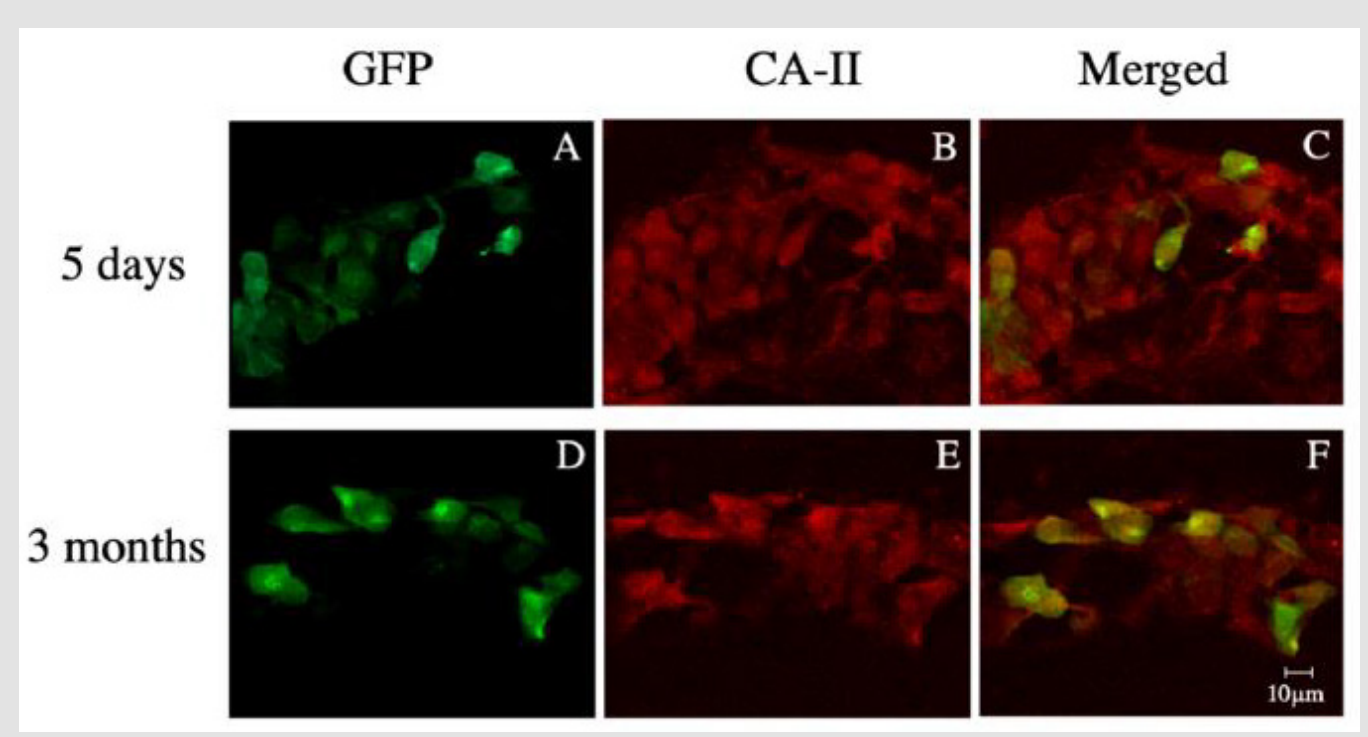

Figure 3: Immunohistochemical analysis of rat brain stained with anti-CAII 5 days

- 5 days (A, B and C) or 3 months (D, E and F) after transduction using the two-step gene transfer method.

- Confocal microscopic images of GFP-positive cells (A and B), CAII-positive cells (C, and D), and merged images (C and F) of the transduced brain tissue sections.

\section{Discussion}

Cell targeting is particularly important for in vivo gene transfer into brain, as stable genetic modification of some neurons or neuronal networks could cause serious psychological changes. Therefore, if the targeted cells are glia, undesirable gene transfer into neurons must be avoided. Our findings show that by using a two-step gene transfer system with Ad and HIV vectors we could selectively transduce OLs both in vitro and in vivo. Moreover, these findings imply that with the appropriate combination of vectors and promoters, one could also selectively transfer genes into neuronal cells.

The transduction efficiency for mixed primary rat brain cells was only $4 \%$ to $5 \%$ (Figure $2 \mathrm{~B}$ ). One likely reason for the low transduction efficiency is that the HIV vector cannot transduce non-human cells, which do not express CXCR4. We therefore had to use two Ad vectors to target rat OLs. On the other hand, only one Ad vector, Ad/MBPCD4, is needed for HIV vector-mediated gene transfer in human brain, which we would expect to increase transduction efficiency. Another possible reason for the low transduction efficiency is the toxicity of Ad vectors [34]. Using Ad vectors it is difficult to achieve highly efficient transgene expression without toxicity [35]. To overcome this problem, to used gutless Ad vectors, which retain no viral genes and have proven to be highly efficient with little toxicity or immunogenicity [36]. In addition, group D Ad reportedly infects primary central nervous system cells more efficiently than group C [37]. Thus, by using recombinant gutless Ad vectors generated from type 17 (group D) Ad, the efficiency of transduction into central nervous system cells using the two-step gene transfer method may be increased.

In summary, we have developed a new method of targeted gene transfer into OLs using Ad vectors with a tissue-specific promoter and an HIV vector. This new method can be used with nondividing cells both in vitro and in vivo. Furthermore, by choosing the appropriate promoters, this method may be useful for in vivo targeted gene transfer into any type of non-dividing cells.

\section{Acknowledgment}

We thank Drs. Ikenaka, Matsushima and Saito for generously providing plasmids and Ad vectors. We also thank Dr. Tsuganezawa for helpful discussion and technical assistance. This work was supported in part by grants from the Ministry of Health and Welfare of Japan and the Ministry of Education, Science and Culture of Japan.

This work was supported by JSPS KAKENHI Grant Number 15K09604 and 18K07859.

\section{Disclosure}

The authors have no conflicts of interest directly relevant to the content of this article. 


\section{References}

1. Edelstein ML, Abedi MR, Wixon J, Edelstein RM (2004) Gene therapy clinical trials worldwide 1989-2004-an overview. J Gene Med 6(6): 597602.

2. Lu Y (2004) Recombinant adeno-associated virus as delivery vector for gene therapy--a review. Stem Cells Dev 13(1): 133-145.

3. Relph KL, Harrington KJ, Pandha H (2005) Adenoviral strategies for the gene therapy of cancer. Semin Oncol 32(6): 573-582.

4. Perry C, Rayat A (2021) Lentiviral Vector Bioprocessing. Viruses 13(2): 268.

5. Gao J, Zhang W, Ehrhardt A (2020) Expanding the Spectrum of Adenoviral Vectors for Cancer Therapy. Cancers (Basel) 12(5): 1139.

6. Baum C (2007) Insertional mutagenesis in gene therapy and stem cell biology. Curr Opin Hematol 14(4): 337-342.

7. Jones RJ, DeBaun MR (2021) Leukemia after gene therapy for sickle cell disease: insertional mutagenesis, busulfan, both, or neither. Blood 138(11): 942-947.

8. Robson T, Hirst DG (2003) Transcriptional Targeting in Cancer Gene Therapy. J Biomed Biotechnol 2003(2): 110-137.

9. Kochergin Nikitsky K, Belova L, Lavrov A, Smirnikhina S (2021) Tissue and cell-type-specific transduction using rAAV vectors in lung diseases. J Mol Med (Berl) 99(8): 1057-1071.

10. Arakawa F, Shibaguchi H, Xu Z, Kuroki M (2002) Targeting of T cells to CEA-expressing tumor cells by chimeric immune receptors with a highly specific single-chain anti-CEA activity. Anticancer Res 22(6C): 42854289.

11. Matsumoto H, Liao S, Arakawa F, Ueno A, Abe H, et al. (2002) Targeting of interleukin-2 to human MK-1-expressing carcinoma by fusion with a single-chain Fv of anti-MK-1 antibody. Anticancer Res 22(4): 2001-2007.

12. Liu X, Tian PK, Ju DW, Zhang MH, Yao M, et al. (2003) Systemic genetic transfer of p21WAF-1 and GM-CSF utilizing of a novel oligopeptidebased EGF receptor targeting polyplex. Cancer Gene Ther 10: 529-539.

13. Belousova N, Krendelchtchikova V, Curiel DT, Krasnykh V (2002) Modulation of adenovirus vector tropism via incorporation of polypeptide ligands into the fiber protein. J Virol 76(17): 8621-8631.

14. Schnierle BS, Stitz J, Bosch V, Nocken F, Merget Millitzer H, et al. (1997) Pseudotyping of murine leukemia virus with the envelope glycoproteins of HIV generates a retroviral vector with specificity of infection for CD4expressing cells. Proc Natl Acad Sci U S A 94(16): 8640-8645.

15. Kowolik CM, Yee JK (2002) Preferential transduction of human hepatocytes with lentiviral vectors pseudotyped by Sendai virus F protein. Mol Ther 5(6): 762-769.

16. Igarashi T, Suzuki S, Takahashi M, Tamaoki T, Shimada T (1998) A nove strategy of cell targeting based on tissue-specific expression of the ecotropic retrovirus receptor gene. Hum Gene Ther 9(18): 2691-2698.

17. Lewis PF, Emerman M (1994) Passage through mitosis is required for oncoretroviruses but not for the human immunodeficiency virus. J Virol 68(1): 510-516.

18. Shimada T, Fujii H, Mitsuya H, Nienhuis AW (1991) Targeted and highly efficient gene transfer into CD4+ cells by a recombinant human immunodeficiency virus retroviral vector. J Clin Invest 88(3): 10431047.

19. Naldini L, Blomer U, Gallay P, Ory D, Mulligan R, et al. (1996) In vivo gene delivery and stable transduction of nondividing cells by a lentiviral vector. Science 272(5259): 263-267.
20. Miyake K, Tohyama T, Shimada T (1996) Two-step gene transfer using an adenoviral vector carrying the CD4 gene and human immunodeficiency viral vectors. Hum Gene Ther 7(18): 2281-2286.

21. Miyake K, Suzuki N, Matsuoka H, Tohyama T, Shimada T (1998) Stable integration of human immunodeficiency virus-based retroviral vectors into the chromosomes of nondividing cells. Hum Gene Ther 9(4): 467475 .

22. Gow A, Friedrich VL, Lazzarini RA (1992) Myelin basic protein gene contains separate enhancers for oligodendrocyte and Schwann cell expression. J Cell Biol 119(3): 605-616.

23. Forghani R, Garofalo L, Foran DR, Farhadi HF, Lepage P, et al. (2001) A distal upstream enhancer from the myelin basic protein gene regulates expression in myelin-forming schwann cells. J Neurosci 21(11): 3780 3787.

24. Miskimins R, Srinivasan R, Marin Husstege M, Miskimins WK, Casaccia Bonnefil P (2002) p27(Kip1) enhances myelin basic protein gene promoter activity. J Neurosci Res 67(1): 100-105.

25. Espinosa de los Monteros A, Sawaya BE, Guillou F, Zakin MM, De Vellis J, et al. (1994) Brain-specific expression of the human transferrin gene. Similar elements govern transcription in oligodendrocytes and in a neuronal cell line. J Biol Chem 269(39): 24504-24510.

26. Ikenaka K, Nakahira K, Nakajima K, Fujimoto I, Kagawa T, et al. (1992) Detection of brain-specific gene expression in brain cells in primary culture: a novel promoter assay based on the use of a retrovirus vector. New Biol 4: 53-60.

27. Kanegae Y, Makimura M, Saito I (1994) A simple and efficient method for purification of infectious recombinant adenovirus. Jpn J Med Sci Biol 47(3): 157-166.

28. Loetscher M, Geiser T, Oreilly T, Zwahlen R, Baggiolini M, et al. (1994) Cloning of a human seven-transmembrane domain receptor, LESTR, that is highly expressed in leukocytes. J Biol Chem 269(1): 232-237.

29. Feng Y, Broder CC, Kennedy PE, Berger EA (1996) HIV-1 entry cofactor: functional cDNA cloning of a seven-transmembrane, $\mathrm{G}$ protein-coupled receptor. Science 272(5263): 872-827.

30. Miyake K, Miyake N, Shimada T (2007) Development of targeted gene transfer into human primary $\mathrm{T}$ lymphocytes and macrophages using high-titer recombinant HIV vectors. J Biotechnol 129(3): 532-538.

31. Paxinos G, Watson C (1986) The Rat Brain in Stereotaxic Coordinates. In: Paxinos G, Watson C (Eds.)., (6 ${ }^{\text {th }}$ Edn.)., Academic Press, New York.

32. Nakano K, Migita M, Mochizuki H, Shimada T (2001) Differentiation of transplanted bone marrow cells in the adult mouse brain. Transplantation 71(12): 1735-1740.

33. Hashimoto M, Aruga J, Hosoya Y, Kanegae Y, Saito I, et al. (1996) A neural cell-type-specific expression system using recombinant adenovirus vectors. Hum Gene Ther 7(2): 149-158.

34. Hsich G, Sena Esteves M, Breakefield XO (2002) Critical issues in gene therapy for neurologic disease. Hum Gene Ther 13(5): 579-604.

35. Franklin R, Quick M, Haase G (1999) Adenoviral vectors for in vivo gene delivery to oligodendrocytes: transgene expression and cytopathic consequences. Gene Ther 6: 1360-1367.

36. Schiedner G, Morral N, Parks RJ, Wu Y, Koopmans SC, et al. (1998) Genomic DNA transfer with a high-capacity adenovirus vector results in improved in vivo gene expression and decreased toxicity. Nat Genet 18(2): 180-183.

37. Chillon M, Bosch A, Zabner J, Law L, Armentano D, et al. (1999) Group $\mathrm{D}$ adenoviruses infect primary central nervous system cells more efficiently than those from group C. J Virol 73(3): 2537-2340. 


\section{ISSN: 2574-1241}

DOI: 10.26717/BJSTR.2021.39.006306

Koichi Miyake. Biomed J Sci \& Tech Res

(C) (P) This work is licensed under Creative

Submission Link: https://biomedres.us/submit-manuscript.php

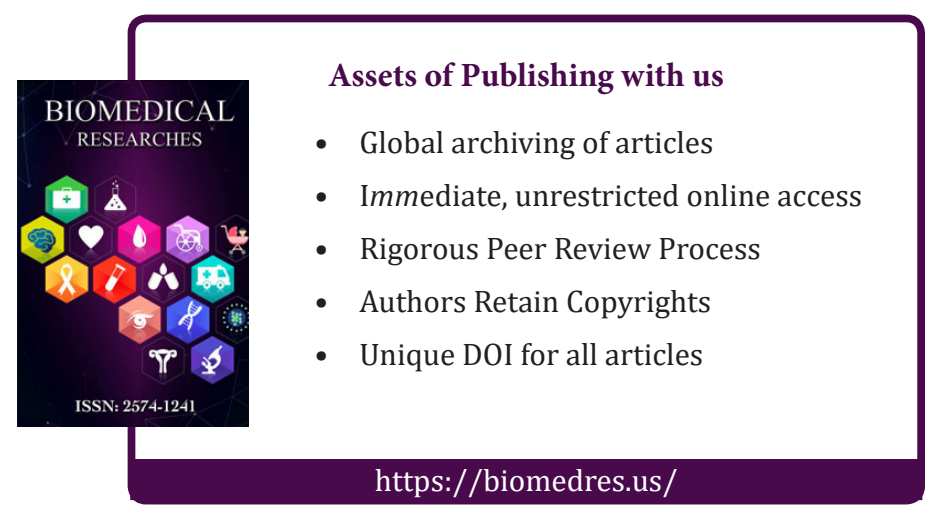

